\title{
Evidence-Based Medicine in Pediatric Endocrinology
}

\author{
Gary E. Butler \\ University College London Hospital and UCL Institute of Child Health, UK
}

\begin{abstract}
This year's evidence-based medicine chapter is different yet again in flavor from last year [1]. Together with large population studies and more RCTs of long-term GH treatment reporting at final height, we have important new evidence in the treatment of preterm infants with thyroxine, definite evidence for the efficacy of insulin pumps and school-based obesity interventions, and clear evidencebased guidance for our endocrine investigation protocols. We see the benefits of $\mathrm{GH}$ on the cardiovascular system in some groups of children, yet warnings about GH use in others. The selection was based on searching under the terms pediatric endocrinology and again under clinical trials. Over 2,000 journal articles were identified from which this selection has been made with the needs of you the clinician in mind.
\end{abstract}

\section{Mechanism of the year \\ Treatment of hypothyroidism in preterm infants}

\section{Phase 1 trial of four thyroid hormone regimens for transient hypothyroxinemia in neonates of $<\mathbf{2 8}$ weeks' gestation}

La Gamma EF, van Wassenaer AG, Ares S, Golombek SG, Kok JH, Quero J, Hong T, Rahbar MH, de Escobar GM, Fisher DA, Paneth N

Department of Neonatal-Perinatal Medicine, Regional Neonatal Center, Maria Fareri Children's Hospital at

Westchester Medical Center, New York Medical College, Valhalla, N.Y., USA

Edmund_lagamma@nymc.edu

Pediatrics 2009; 124:e258-268

Background: Transiently low levels of thyroid hormones have been shown to be associated with higher rates of cerebral palsy and cognitive impairment in approximately $50 \%$ of neonates born $24-28$ weeks' gestation. This study aimed to identify whether any of four thyroid hormone supplementation regimens could raise $\mathrm{TT}_{4}$ and $\mathrm{FT}_{4}$ levels and improve neurodevelopmental outcome without suppressing TSH (biochemical euthyroidism).

Methods: Eligible subjects had gestational ages between 24 and 28 weeks, and were entered into a masked randomized trial $<24 \mathrm{~h}$ of birth to one of six study arms ( $\mathrm{n}=20-27$ per arm): placebo (vehicle: $5 \%$ dextrose), potassium iodide $(30 \mu \mathrm{g} / \mathrm{kg} / \mathrm{day})$ and continuous or bolus daily infusions of either $4 \mathrm{or} 8 \mu \mathrm{g} /$ $\mathrm{kg} /$ day of $\mathrm{T}_{4}$ for 42 days. $1 \mu \mathrm{g} / \mathrm{kg} /$ day of $\mathrm{T}_{3}$ was added during the first 14 postnatal days to the $\mathrm{T}_{4}$ and was infused with $1 \mathrm{mg} / \mathrm{ml}$ albumin to prevent adherence to plastic tubing.

Results: $\mathrm{FT}_{4}$ levels were high in the first 7 days in all hormone-treated subjects, however only the treatment study limb which showed a significant elevation in all treatment periods was continuously $8 \mu \mathrm{g} / \mathrm{kg} /$ day ( $\mathrm{p}<0.002$ vs. all other groups). $\mathrm{TT}_{4}$ remained elevated in the first 7 days in all hormone-treated subjects ( $\mathrm{p}<0.05$ vs. placebo or iodine arms). After 14 days, both $8 \mu \mathrm{g} / \mathrm{kg} / \mathrm{day}$ arms as well as the continuous $4 \mu \mathrm{g} / \mathrm{kg} /$ day arm produced a sustained elevation of the mean and median $\mathrm{TT}_{4},>7 \mu \mathrm{g} / \mathrm{dl}(90 \mathrm{~nm} / \mathrm{l} ; \mathrm{p}<0.002$ vs. placebo). The lowest suppression of TSH occurred during the continuous infusion of $4 \mu \mathrm{g} / \mathrm{kg} /$ day $\mathrm{T}_{4}$ arm. Unexpectedly the duration of mechanical ventilation was significantly lower in the continuous $4 \mu \mathrm{g} / \mathrm{kg} / \mathrm{day} \mathrm{T}_{4}$ arm and in the bolus $8 \mu \mathrm{g} / \mathrm{kg} / \mathrm{day} \mathrm{T}_{4}$ arm $(\mathrm{p}$ $<0.05$ vs. remaining arms). Retinopathy of prematurity was significantly lower in all the four thyroid hormone treatment arms compared with the combined placebo and iodine arms $(\mathrm{p}<0.04)$. The incidence of necrotizing enterocolitis was higher in the combined $8 \mu \mathrm{g} / \mathrm{kg} / \mathrm{day}$ arms $(\mathrm{p}<0.05$ vs. other arms). 
Conclusion: Continuous supplement of low-dose thyroid hormone $(4 \mu \mathrm{g} / \mathrm{kg} / \mathrm{day})$ for 42 days caused elevation of $\mathrm{TT}_{4}$ with only a modest suppression of TSH. This was associated with several improved clinical parameters suggesting that $\mathrm{T}_{4}$ was of benefit. Future trials will be needed to evaluate the long-term neurodevelopmental effects of such supplementation.

Because extremely preterm low-birthweight infants frequently suffer severe systemic illness and experience changing biological requirements during a period of critical brain development, using hormone values based either healthy fetal levels or levels observed in term neonates to determine thyroid status is probably inappropriate, and in any case does not give any guidance in the need for $\mathrm{T}_{4}$ replacement in the transient hypothyroidism of prematurity. The authors' view is that biochemical euthyroidism is the minimum thyroid hormone threshold associated with a reduced risk of neonatal illness or better long-term neurodevelopment. This study was an elegantly designed masked randomized trial investigating two $\mathrm{T}_{4}$ dosage schedules in different delivery modes (bolus or continuous), with or without initial $\mathrm{T}_{3}$ co-supplementation and designed to avoid overtreatment which has also been reported as harmful. The goal for a minimum threshold for $\mathrm{FT}_{4}$ concentration was identified as $19 \mathrm{pmol} / \mathrm{l}$, for $\mathrm{TT}_{4}$ concentration as $77 \mathrm{nmol} / \mathrm{l}$, and for $\mathrm{TT}_{3}$ concentration as $0.8 \mathrm{nmol} / \mathrm{l}$. Supplementation of the prohormone $T_{4}$ with the active hormone $T_{3}$ for the first 14 postnatal days raised the $\mathrm{TT}_{3}$ blood level but was depressed by TSH values to or below the assay's level of detection, indicating overtreatment and suggesting that $\mathrm{T}_{3}$ supplementation is unnecessary. In addition, the $8 \%$ mortality rate in the two $4 \mu \mathrm{g} / \mathrm{kg} / \mathrm{day}$ arms was half that of the placebo or iodine arms (16\%) and also less than half that of higher dosage arms (22\%). Furthermore, a statistically significant reduction in retinopathy of prematurity was found compared with the iodine/placebo arms. The authors describe this as intriguing, meriting future investigation, as thyroid hormone effects on vascular and retinal development in both animals and humans have been described. This trial accomplished the goal of elevating thyroid hormone blood levels in extremely premature neonates to exceed a predefined target threshold without completely suppressing TSH. This was most successfully achieved by using continuous $4 \mu \mathrm{g} / \mathrm{kg} / \mathrm{day} \mathrm{T}_{4}$ as replacement therapy.

New paradigms

Patches versus pills

\section{Conjugated oral versus transdermal estrogen replacement in girls with Turner syndrome: a pilot comparative study}

Nabhan ZM, Dimeglio LA, Qi R, Perkins SM, Eugster EA

Section of Pediatric Endocrinology/Diabetology, Indiana University School of Medicine, Riley Hospital for Children,

Indianapolis, Ind., USA

znabhan@iupui.edu

J Clin Endocrinol Metab 2009;94:2009-2014

Background: This study aimed to compare conjugated oral versus transdermal estrogen (TD E2) on bone accrual, uterine growth, pubertal development, IGF-1, and lipids in Turner syndrome (TS) as the optimal route of estrogen delivery is unknown.

Methods: Twelve prepubertal GH-treated girls aged $14.0 \pm 1.7$ years were randomized to conjugated oral estrogen or TD E2. The study protocol evaluated changes in dual-emission x-ray absorptiometry, pelvic ultrasound, Tanner staging, growth velocity, IGF-1, and lipid profile at 6 monthly intervals over 1 year.

Results: TD E2 therapy resulted in a significantly greater change in spine bone accrual parameters at 12 months compared with conjugated oral estrogen (bone mineral content $9.0 \pm 0.9$ vs. $5.8 \pm 0.9 \mathrm{~g}, \mathrm{p}=$ 0.04 ; bone mineral density $0.12 \pm 0.01$ vs. $0.06 \pm 0.01 \mathrm{~g} / \mathrm{cm}^{2}, \mathrm{p}=0.004$; Z-score $0.7 \pm 0.1$ vs. $0.3 \pm 0.1$, $\mathrm{p}=0.03$ ). TD E2 also produced better uterine growth than the oral group at 1 year (length $4.13 \pm 0.39$ vs. $1.98 \pm 0.39 \mathrm{~cm}, \mathrm{p}=0.003$; volume $22.2 \pm 4.4$ vs. $4.0 \pm 4.4 \mathrm{ml}, \mathrm{p}=0.02$ ). After 12 months, $66 \%$ of subjects in the TD group had a mature uterus compared with $0 \%$ in the oral group. No other significant differences were seen. 
Conclusion: In girls with TS, this pilot study showed that TD E2 resulted in faster bone accrual in the spine and increased uterine growth compared with conjugated oral estrogen. This preliminary information for optimizing estrogen replacement in this population may inform further large-scale, long-term studies.

Still much controversy surrounds the choice of estrogen preparation to induce puberty in girls with TS and by inference in chromosomally normal hypogonadal girls. The authors acknowledge that this is a small-scale pilot study, but the reason for inclusion here is that it is the first prospective randomized controlled trial comparing conjugated oral versus TD E2 for pubertal induction to be performed. Conjugated oral estrogen was administered at a dose of $0.3 \mathrm{mg}$ every day for the first 6 months followed by $0.3 \mathrm{mg}$ alternating with $0.625 \mathrm{mg}$ every day for the second 6 months, whereas the TD E2 group was treated with a 0.025 -mg patch twice a week for 6 months followed by a 0.0375 -mg patch twice a week for the second 6 months. These doses were chosen based on published equivalences. The significant apparent benefit of TD E2 on more rapid uterine maturation and bone accrual could be as a result of incorrect assumptions as to the bioequivalent of each estrogen preparation, but no differences in height velocity, IGF-1, lipid parameters or degree of breast development attained between the two groups were reported. It is recognized that synthetic estrogens (ethinyl estradiol) are also frequently used for the induction of puberty in TS, the authors discuss publications which cite a lack of evidence for a difference on bone mineralization between the different oral preparations, but that TD E2 had improved bone mineral density in young adult patients. This pilot study provides a good evidence base and platform for larger scale research programs to confirm that TD E2 appears to be a significantly better modality for estrogen replacement in TS and potentially other hypogonadal girls.

\section{New hope \\ Do we now have the evidence base for insulin pumps?}

\section{Continuous subcutaneous insulin infusion (CSII) versus multiple insulin injections for type 1 diabetes mellitus}

Misso ML, Egberts KJ, Page M, O'Connor D, Shaw J

Australasian Cochrane Centre, Monash Institute of Health Services Research, Monash University, Clayton, Vic., Australia

Cochrane Database Syst Rev 2010;CD005103

Background: This review aimed to assess the effects of continuous subcutaneous insulin infusion (CSII) compared to multiple insulin injections (MI) in people with type 1 diabetes mellitus. The onset of type 1 diabetes mellitus may occur at any age and it is one of the commonest chronic diseases of childhood and adolescence. Since there are currently no known interventions to prevent onset, it is vital that effective treatment regimes are available.

Methods: Electronic searches were made of The Cochrane Library, MEDLINE, EMBASE and CINAHL. Studies were included if they were randomized controlled trials comparing CSII with three or more insulin injections per day (MI) in people with type 1 diabetes mellitus. They were subsequently assessed by two authors who independently evaluated the risk of bias and extracted characteristics of selected studies. Study investigators were contacted to obtain missing information. Generic inverse variance meta-analyses using a random-effects model were performed.

Results: 23 studies randomized 976 participants with type 1 diabetes to either intervention. There was a statistically significant difference in glycosylated hemoglobin A1c (HbA1c) favoring CSII (weighted mean difference $-0.3 \%$ (95\% CI interval -0.1 to -0.4$)$. There were no obvious differences between the interventions for non-severe hypoglycemia, but severe hypoglycemia appeared to be reduced in those using CSII. Quality of life measures suggest that CSII is preferred over MI. No significant difference was found for weight. Adverse events were not well reported, no information is available on mortality, morbidity and costs. 
Conclusion: There is some evidence to suggest that CSII may be better than MI for glycemic control in people with type 1 diabetes. Non-severe hypoglycemic events do not appear to be reduced with CSII. There is insufficient evidence regarding adverse events, mortality, morbidity and costs.

The Health Technology Assessment report and the National Institute for Clinical Excellence (NICE) guidelines on the use of CSII are methodologically rigorous, however the most up-to-date search strategies were conducted in 2007 and therefore do not include recent randomized controlled trials [2]. Given the limitations of these previous systematic reviews, this review has considered these factors and used specific methodology and criteria outlined by the Cochrane Collaboration in their intent to present a comprehensive systematic review to assess the effects of CSII compared to MI. Meta-analyses were performed where possible and feasible. There may be benefit in using CSII over MI for improving glycaemic control and improving health-related quality of life in people with type 1 diabetes. Non-severe hypoglycemic events do not appear to be different between either treatment methodology. It is important to note that there is insufficient evidence regarding adverse events, reductions of diabetes late complications, mortality and cost which are all vital in deciding which treatment regimen to choose. Until evidence is available for these outcomes, firm recommendations as to whether CSII is superior in the management of people with type 1 diabetes are not possible. Long-term, large-scale and methodologically rigorous studies are needed to determine the effect of CSII and MI on outcomes such as hypoglycemia, mortality, diabetes late complications and other adverse effects, using validated scales. Cost-effectiveness data are also needed to support choice and for healthcare budgeting purposes.

\section{New concerns}

\section{Increased risk of thyroid pathology in patients with thyroid hemiagenesis: results of a large cohort case-control study}

Ruchala M, Szczepanek E, Szaflarski W, Moczko J, Czarnywojtek A, Pietz L, Nowicki M, Niedziela M, Zabel M, Kohrle J, Sowinski J

Department of Endocrinology, Metabolism and Internal Medicine, University of Medical Sciences, Poznan, Poland mruchala@ump.edu.pl

Eur J Endocrinol 2010;162:153-160

Background: Thyroid hemiagenesis (THA) is an anomaly resulting from the developmental failure of one lobe of the thyroid. The etiology, clinical significance, and management of patients in whom THA is identified are still unclear. The aim of the study is to provide the first controlled systematic analysis of 40 patients with THA in comparison with 80 subjects with a fully developed thyroid gland out of 2,159 participants from a cross-sectional population-based thyroid screening program.

Methods: Serum TSH, free thyroxine $\left(\mathrm{FT}_{4}\right)$, free triiodothyronine $\left(\mathrm{FT}_{3}\right)$, and thyroid autoantibodies were measured in all subjects. In 37 of the THA patients, thyroid ultrasonography and a ${ }^{99 \mathrm{~m}} \mathrm{Tc}$ thyroid scintiscan were performed, followed by fine-needle aspiration biopsy if indicated. The remaining archival 3 cases were diagnosed with the use of ${ }^{131}$ I scintiscan pre- and post-TSH stimulation.

Results: In this study, a considerable prevalence of women with THA was noted, with 7:1 female-to-male ratio. Of the 40 patients, 35 had left-sided agenesis. Patients with THA were usually clinically euthyroid but levels of TSH and $\mathrm{FT}_{3}$ were significantly higher, with a higher $\mathrm{FT}_{3} / \mathrm{FT}_{4}$ ratio in comparison with the controls. Furthermore, a higher incidence of functional, morphological, and autoimmune thyroid disorders was seen in THA patients compared controls $(\mathrm{p}<0.05)$.

Conclusion: This study showed that individuals with THA are more likely to develop thyroid pathology. This may be as a result of the long-lasting TSH overstimulation. The diagnosis of THA should be followed by systematic observation and adequate levothyroxine treatment in patients with elevated TSH level.

This paper provides a long-term outlook in patients with a rare abnormality of the thyroid gland. The prevalence of THA is estimated to be $0.2-0.025 \%$. Although conducted in a moderately iodine- 
deficient area of Poland, this study was of a prospective design with an age- and sex-matched control group. In 17/40 the anomaly was detected by screening or incidentally, all controls being recruited from the screening program. The study looked at patients between 12 and 79 years of age, so, from the pediatric perspective, is able to give us an idea of long-term prognosis if THA is identified, as in this study via a population screening program. However, the younger patients ( $<25$ years) were less likely to have compensatory hypertrophy of the residual thyroid lobe and a lower incidence of autoimmune disease compatible with the congenital etiology. Older patients were more likely to have autoimmune disease and compensatory hypertrophy. THA was more frequently associated with hyperthyroidism and higher TSH levels and thus indicating levothyroxine replacement. In the 22 patients who had fine-needle aspiration biopsy of suspicious lesions on ultrasound scan, no malignancies were found, even though 6 proceeded to surgery due to uncertainties on the initial biopsy or goitre size. In the absence of a clear genetic cause for THA, even in familial cases ongoing follow-up is indicated in these patients. The debate also continues as to the upper normal level for plasma TSH and whether earlier levothyroxine substitution may alter outcome in some patients with this condition.

\section{Concepts revised \\ The age of adolescence}

\section{Recent decline in age at breast development: the Copenhagen Puberty Study}

Aksglaede L, Sorensen K, Petersen JH, Skakkebaek NE, Juul A

Rigshospitalet, Department of Growth and Reproduction, Copenhagen, Denmark

lise.aksglaede@rh.hosp.dk

Pediatrics 2009;123:e932-939

Background: Data published showing an earlier onset of breast development in American girls, NHANES III, has been controversial. However, secular trend analyses are often limited by poor comparability between studies over different time periods and counties. This study presents new European data systematically collected from the same region and by the same research group at the beginning over a 15 -year period up to the present.

Methods: 2,095 girls aged 5.6-20.0 years were studied in 1991-1993 (1991 cohort; $\mathrm{n}=1,100)$ and 2006-2008 (2006 cohort; $n=995$ ). Puberty was staged by palpation of glandular breast tissue, and measurement of height and weight, and blood sampling for estradiol, luteinizing hormone, and folliclestimulating hormone was done. Age at entering pubertal breast stages 2-5, pubic hair stages 2-5, and menarche was documented for the two cohorts.

Results: The onset of puberty (Tanner breast stage 2+) occurred significantly earlier in the 2006 cohort (estimated mean age 9.86 years) compared with the 1991 cohort (estimated mean age 10.88 years). This difference remained significant after adjustment for BMI, thus excluding obesity as a variable. Estimated ages at menarche were 13.42 and 13.13 years in the 1991 and 2006 cohorts, respectively. Serum FSH and LH did not differ between the two cohorts at any age interval. However, estradiol levels were significantly lower in 8- to 10-year-old girls from the 2006 cohort in comparison with girls of the same age from the 1991 cohort.

Conclusion: This study demonstrated significantly earlier breast development but less of a reduction in the age at menarche among a recent population of girls compared with a similar one 15 years earlier. Variation in reproductive hormones and in BMI did not explain these marked changes. Other factors yet to be identified may be involved. 


\section{Recent changes in pubertal timing in healthy Danish boys: associations with body mass index}

Sorensen K, Aksglaede L, Petersen JH, Juul A

Department of Growth and Reproduction, Copenhagen University Hospital, Copenhagen, Denmark

kaspar.Soerensen@rh.regionh.dk

J Clin Endocrinol Metab 2010;95:263-270

Background: The American population-based study, NHANES III, identified possible secular trends in male puberty in the 1990s. However, no conclusions could be made about the age of the onset of puberty due to the lack of comparable data. This study aimed to evaluate secular trends in pubertal onset during the recent 15 years and their relation to body mass index (BMI) in boys.

Methods: This was a cross-sectional study in 1991-1993 and a combined cross-sectional and longitudinal study in 2006-2008 (The Copenhagen Puberty Study). 1,528 boys aged 5.8-19.9 years were studied in 1991-1993 (1991 cohort; $\mathrm{n}=824$ ) and 2006-2008 (2006 cohort; $\mathrm{n}=704$ ) at a tertiary centre for pediatric endocrinology. Genital and pubic hair stages as well as testicular volume using an orchidometer were evaluated. Blood samples were analyzed for LH, FSH, testosterone, and SHBG.

Results: The mean age of the onset of puberty (attainment of testicular volume $>3 \mathrm{ml}$ ) of 11.66 years (95\% CO 11.49-11.82) occurred significantly earlier in 2006-2008 than in the 1991-1993 cohort, 11.92 years $(11.76-12.08) ; p=0.025$. Significantly higher LH levels were found in the 11- to 16-yearold boys from 2006-2008 compared with 1991-1993 ( $\mathrm{p}=0.020)$, but this was not found with testosterone. The BMI Z-score increased significantly from $0.044(-0.016$ to 0.104$)$ in $1991-1993$ to 0.290 $(0.219-0.361)$ in 2006-2008; $\mathrm{p}<0.001$. Interestingly, pubertal onset and LH levels did not differ significantly between study periods after adjustment for BMI.

Conclusion: Estimated mean age at the onset of puberty has declined significantly over the past 15 years. This decline was associated with the coincident increase in BMI.

Knowledge about the timing of sexual development in children and adolescents and the causes and consequences is fundamentally important not only for the practice of medicine but in society as well. Although a series of publications from the USA and other European countries had suggested a lowering of the age of the onset of puberty, there is a consistent finding that the tempo or transit time through the pubertal process is not necessarily accelerating at the same pace [3]. This is evident in this study at least in girls with a reduction in the age at menarche by only 0.3 years whereas age at B2 is 1 year earlier. There are different tempo patterns emerging between boys and girls too. The age of B2 is shifted to the left across the whole age spectrum, whereas earlier pubertal development in boys is limited to those beginning before age 12.5 years. Thus the latter $20 \%$ of boys entering puberty do not seem to show this secular trend. Contrary to popular myth, obesity does not appear to be implicated in these trends, the BMI effect cancels itself out in girls when controlled for, and the early developers in boys are not the ones showing a rise in BMI; paradoxically, boys going into puberty later are the ones where BMI rises, and there is no secular trend here as discussed above. The relationship between reproductive hormones and clinical pubertal changes is important to mention as the earlier physical changes were not matched by rises in gonadotrophins or sex hormones. Paradoxically, testosterone and estradiol levels were lower at maturity in the recent compared with the historical cohorts. Clearly there is much need for further exploration and research, but the value of this study is its carefully designed methodology and attempt to find the evidence base upon which we can improve our knowledge of childhood and develop our clinical practice. The picture is clearly more complicated than was expected. 


\section{The glucagon test in the diagnosis of growth hormone deficiency in children with short stature younger than 6 years}

Secco A, di lorgi N, Napoli F, Calandra E, Ghezzi M, Frassinetti C, Parodi S, Casini MR, Lorini R, Loche S, Maghnie M Department of Pediatrics, Istituto di Ricovero e Cura a Carattere Scientifico G Gaslini Institute, University of Genova, Genova, Italy

J Clin Endocrinol Metab 2009;94:4251-4257

Background: The aim of this study, conducted in two pediatric endocrinology centers, was to investigate the diagnostic value of the glucagon test as an alternative to insulin tolerance test (ITT) and arginine test in children younger than 6 years with GHD as few studies have addressed the diagnostic role of the glucagon test in children when GH deficiency (GHD) is suspected.

Methods: 48 children (median age 4.2 years and median height -3.0 SD score) with GHD confirmed by a peak GH to ITT and arginine $<10$ and (median 4.7 and $3.4 \mu \mathrm{g} / \mathrm{l}$ respectively) subsequently underwent a glucagon stimulation test. Magnetic resonance imaging had showed normal hypothalamic-pituitary anatomy in 24 children, isolated anterior pituitary hypoplasia in 7, and structural hypothalamic-pituitary abnormalities in 17.

Results: The median GH peak response to glucagon $(13.5 \mu \mathrm{g} / \mathrm{l})$ was significantly higher than that observed after ITT and arginine ( $<$ 0.0001). 20 subjects (group 1) had a GH peak after glucagon which was $<10 \mu \mathrm{g} / \mathrm{l}$ and 28 subjects (group 2) had peak GH levels of $>10 \mu \mathrm{g} / \mathrm{l}$ without significant clinical or biochemical differences between the two groups. Median GH peak after glucagon was similar between patients with multiple pituitary hormone deficiency and those with isolated GHD and between subjects with and without structural hypothalamic-pituitary abnormalities. There was a negative correlation between age at diagnosis and the magnitude of the GH peak after glucagon $(r=-0.636, p<0.0001)$.

Conclusion: Glucagon can effectively release GH and can be used to evaluate somatotroph function in young children with short stature. However, the differing results between GH levels and those following ITT and arginine in this study suggest that normative data for this test in young children need to be established.

\section{Reassessment of the growth hormone status in young adults with childhood-onset growth hormone deficiency: reappraisal of insulin tolerance testing}

Secco A, di lorgi N, Napoli F, Calandra E, Calcagno A, Ghezzi M, Frassinetti C, Fratangeli N, Parodi S, Benassai M, Leitner Y, Gastaldi R, Lorini R, Maghnie M, Radetti G

Department of Pediatrics, Instituto di Ricovero e Cura a Carattere Scientifico, Giannina Gaslini Institute, University of Genova, Genova, Italy

J Clin Endocrinol Metab 2009;94:4195-4204

Background: The aim of this study, performed in two pediatric endocrinology centers, was to evaluate the accuracy of the insulin tolerance test (ITT), mean 12-h spontaneous nocturnal GH (SNGH), and IGF-1 in the diagnosis of permanent GH deficiency (GHD) in young adults with childhood-onset GHD. The 2007 Consensus Statement had suggested a peak GH to the ITT of $<6 \mu \mathrm{g} / \mathrm{l}$ as being the diagnostic cutoff for permanent GHD, although further validation was recommended.

Methods: ITT, 12-h SNGH, and IGF-1 were evaluated as single or combined tests in 79 subjects with childhood-onset GHD (median age, 18.0 years). 48 of the subjects had isolated GHD or one additional pituitary defect and normal MRI or anterior pituitary hypoplasia (low likelihood GHD group), and 31 subjects had structural hypothalamic-pituitary abnormalities or multiple pituitary hormone deficiencies (high likelihood GHD). Diagnostic criteria based on the cut-offs identified by ROC analysis were combined, both in series (i.e. considering as positive a subject who was positive on each test) and in parallel (i.e. considering as positive a subject who was positive on at least one test).

Results: Receiver operating characteristic curve analysis showed the most accurate parameters for the diagnosis of GHD was a peak GH in the ITT of $5.6 \mu \mathrm{g} / \mathrm{l}$ or lower (sensitivity $77 \%$; specificity $94 \%$; area under the curve $($ AUC) $=0.92$ ), mean 12 -h SNGH of $1.20 \mu \mathrm{g} / \mathrm{l}$ or lower (sensitivity $90 \%$; specificity 
$90 \%$; AUC $=0.93$ ), and IGF-1 of -2.8 SD score or lower (sensitivity $81 \%$; specificity $96 \%$; AUC = 0.93). Seven patients in the high likelihood GHD group showed a peak GH to ITT $>5.6 \mu \mathrm{g} / \mathrm{l}$, but a median IGF-1 that was significantly lower than that of group low likelihood GHD $(-3.3$ vs. $-0.7 \mathrm{SD}$ score; $\mathrm{p}=0.0001$ ). Peak GH to ITT of $3.6 \mu \mathrm{g} / \mathrm{l}$ or lower and to arginine of $3.1 \mu \mathrm{g} / \mathrm{l}$ or lower at childhood diagnosis can predict a future permanent GHD condition.

Conclusion: Using a cut-off point of a peak GH to ITT $<5.6 \mu \mathrm{g} / \mathrm{l}$ performs well as an accurate diagnostic strategy for determining persistent GHD in young adults with childhood-onset GHD. In addition, IGF-1 is a reliable marker providing information about the severity of GHD. Careful follow-up is needed for subjects who have discordant ITT and IGF-I results.

Despite decades of reliance on GH stimulation tests, their use is highly problematic as discussed in the past and pointed out in this pair of papers [4]. Among the various GH stimulation tests, the glucagon test has been poorly studied in the pediatric population. In a systematic study investigating patients already confirmed as GHD on two other stimulation tests, there was a surprise finding of the GH peak after glucagon being $>10 \mu \mathrm{g} / \mathrm{l}$ in 28 of 48 patients $(58.3 \%), 15$ (35.4\%) of whom had abnormal anatomy on MRI scanning and/or MPHD. Therefore, by standard criteria, 28 of the patients would have been diagnosed as normal. In this study, the mean GH response to glucagon was higher than that observed after ITT or arginine. This could suggest that the GH-releasing effect of glucagon in young children with congenital GHD is greater than that of ITT and arginine, and thus, the same cut-off levels cannot be adopted. There was also an association between a very low IGF-1 concentration with a GH peak of $<10 \mu \mathrm{g} / \mathrm{l}$ after both ITT and arginine. The authors caution that GH responses to glucagon may give false-negative results when the threshold of normal of 10 or $20 \mu \mathrm{g} / \mathrm{l}$ is used. In the absence of normative data for $\mathrm{GH}$ responses to pharmacological stimuli in this age group, this reinforces the caution to exert in the interpretation of GH stimulation tests.

In contrast, data on re-evaluation of young adults with childhood-onset GHD provides the evidence for a good diagnostic performance for a peak GH cut-off level of $<5.6 \mu \mathrm{g} / \mathrm{l}$ in the diagnosis of GHD. The presence of anatomical abnormalities on MRI, together with two or more pituitary hormone deficiencies, was taken as the basis for the prediction of diagnostic accuracy. ROC curve analysis indicated the best diagnostic accuracy for a peak GH to ITT of $5.6 \mu \mathrm{g} / \mathrm{l}$ with an AUC of 0.92 , demonstrating that ITT is a reliable test for evaluation of GH secretion and confirming that the GH peak adopted by the 2007 Consensus is adequate for the definition of permanent GHD in young adults [5]. Measurement of IGF-I provides useful information in direct parallel to the degree of GHD. ROC analysis of IGF-1 showed the best diagnostic accuracy for an IGF-1 cut-off of -2.8 SDS with $96 \%$ specificity. Specificity of GH peak and IGF-1 levels are similar, suggesting that a single IGF-1 evaluation may have a role in the definition of GH status, but that normal IGF-1 concentrations do not exclude GHD in approximately $20 \%$ of young adult patients. Overnight measurement of spontaneous $\mathrm{GH}$ secretion was not determined to be cost-effective, either as a diagnostic test or for economic reasons. The authors did not find that clinical parameters at the time of initial diagnosis during childhood played any role in the prediction of GH status after adult height attainment, whereas ROC analysis applied to $\mathrm{GH}$ peaks obtained during childhood showed that a peak $\mathrm{GH}$ of about $3 \mu \mathrm{g} / \mathrm{l}$ after at least one stimulation test offered good accuracy for the discrimination of patients with a high likelihood of GHD in adult life from those who might subsequently normalize GH secretion. However, this predictive accuracy could be overestimated because it was based on observations of a cut-off point derived from retrospective data. One limitation of these analyses is that $\mathrm{GH}$ assay used in many previous studies is now obsolete.

\section{Precocious pubarche: distinguishing late-onset congenital adrenal hyperplasia from premature adrenarche}

Armengaud JB, Charkaluk ML, Trivin C, Tardy V, Breart G, Brauner R, Chalumeau M

Université Paris Descartes, Paris, France

J Clin Endocrinol Metab 2009;94:2835-2840

Background: In 5-20\% of children presenting with precocious pubarche (PP), late-onset congenital adrenal hyperplasia (LO-CAH) is found and an adrenal stimulation test is recommended in the diagnostic schedule, but the results are normal in more than $80 \%$ of cases and it is stressful and expensive, so the aim was to identify clinical and plasma predictors of LO-CAH among patients presenting with PP. 
Methods: This was a retrospective cohort study that included all patients seen for PP between 1999 and 2006 ( $\mathrm{n}=238$ ). All underwent an ACTH test. LO-CAH was diagnosed by a post-ACTH 17-hydroxyprogesterone $(17-\mathrm{OHP})$ plasma level $>10 \mathrm{ng} / \mathrm{ml}(30 \mathrm{nmol} / \mathrm{l})$ and confirmed by mutational analysis of the CYP21 gene.

Results: No statistically significant difference was observed between the distributions of the age at pubic hair onset, weight, height, and pubic hair Tanner stage at the time of the first medical examination in patient with LO-CAH and PA. Basal levels of $17-\mathrm{OHP}, \Delta^{4}$-androstenedione, and testosterone plasma levels were significantly higher in the 10 of $238(4 \%)$ patients who had LO-CAH. A $2 \mathrm{ng} / \mathrm{ml}(6 \mathrm{nmol} / \mathrm{l})$ threshold for basal 17-OHP plasma levels offered 100\% (95\% CI 69-100) sensitivity for the diagnosis of LO-CAH and 99\% (95\% CI 96-100) specificity.

Conclusion: Although the three above factors were predictive of LO-CAH, 17-OHP was the most efficient. Applying a selective approach using a $2-\mathrm{ng} / \mathrm{ml}(6 \mathrm{nmol} / \mathrm{l})$ basal $17-\mathrm{OHP}$ plasma level cut-off would have safely avoided $99 \%$ of the unnecessary ACTH tests in this patient group.

Although a retrospective audit rather than primary research, this is the largest and most detailed study addressing this common diagnostic conundrum. The study examined clinical, basal and stimulated hormonal measures for their performance in their ability to predict LO-CAH. Presentation of clinical features such as age, sex, degree of pubic hair development did not show significant differences, although LO-CAH patients tended to have a higher BMI than those with PP. Basal 17-OHP was superior in its discriminating power (area under the ROC curve of 0.99), performing significantly better than androstenedione $(0.90)$, testosterone $(0.83)$ and the most discriminatory clinical parameter BMI (0.66) for comparison. Stimulated 17-OHP levels actually did not add further clarification of the diagnosis which was confirmed in all cases with mutations in the CYP21 gene. In this time of a global recession, we are increasingly being asked to look at efficiency measures in our daily clinical practice. This paper provides a good evidence base for a re-evaluation of practice. The diagnosis of LO-CAH can be made confidently with basal 17-OHP level, without the need for a stimulation test or additional hormonal analyses. If the study is confirmed on a higher number of affected individuals (only 10 here), this will allow savings in laboratory and drug costs and be better for the patient as hospital admission and a stimulation test can be avoided. One important point not discussed in the paper is the timing of basal 17-OHP measurement due to the circadian rhythm of 17-OHP secretion and risk of false negative result if blood is drawn later in the day.

\section{Association of thyroid gland volume, serum insulin-like growth factor-l, and anthropometric variables in euthyroid prepubertal children}

Boas M, Hegedus L, Feldt-Rasmussen U, Skakkebaek NE, Hilsted L, Main KM

University Department of Growth and Reproduction, Rigshospitalet, Copenhagen, Denmark

malene.boas@rh.regionh.dk

J Clin Endocrinol Metab 2009;94:4031-4035

Background: This study investigated thyroid gland volume and several hormonal and anthropometric variables in prepubertal children as previous studies had focused on the interrelation between thyroid size, anthropometric variables, and IGF-I in adults, but data such as this was lacking in children.

Methods: 859 prepubertal euthyroid Danish children aged 4-9 years underwent a thorough clinical investigation, including anthropometrical measurements. Additionally, determination of TSH, thyroid hormones, autoantibodies, urinary iodine excretion was estimated, and thyroid volume (TV) measured by ultrasound. Longitudinal growth data from birth were available.

Results: TV increased significantly with age $(\mathrm{r}=0.487 ; \mathrm{p}<0.001)$. Mean TV \pm SD for different age groups were as follows: 4 years, $2.2 \pm 1.4 \mathrm{ml}$; 5 years, $2.5 \pm 1.3 \mathrm{ml} ; 6$ years, $2.8 \pm 1.3 \mathrm{ml} ; 7$ years, $3.2 \pm 1.3 \mathrm{ml} ; 8$ years, $3.5 \pm 1.3 \mathrm{ml}$; 9 years, $3.7 \pm 1.3 \mathrm{ml}$. We found a significant positive association between IGF-1 and TV $(\mathrm{p}<0.001)$. Moreover, in multiple regression analyses, TV correlated significantly with TSH $(\mathrm{p}<$ $0.013)$, free $\mathrm{T}_{4}(\mathrm{p}<0.001)$, lean body mass $(\mathrm{p}<0.001)$, and body surface area $(\mathrm{p}<0.001)$ as well as other anthropometrical measurements. TV also showed a positive correlation with family history of thyroid disease and presence of incidental abnormal ultrasound findings ( $\mathrm{p}=0.025$ and 0.022 , respectively).

Conclusion: In this study of prepubertal Danish children, the GH/IGF-1-axis was positively correlated with thyroid size, suggesting that it may have a role in the regulation of thyroid growth. Additionally, anthropometric measurements, especially body surface area, were the best predictors of TV. 
This large-scale population cohort study was able to define factors associated with thyroid gland growth, mainly by inference and comparison with other anthropometric variables and serum markers. Thyroid volume highly significantly correlated with age and anthropometric parameters: height, weight, BSA, BMI, and estimated lean body mass. Body size was strongly associated with age, but even when controlling for age by using SDS, all anthropometrical variables were positively associated with thyroid volume SDS. These positive correlations are reassuring and reassuring that in normal children the thyroid gland grows as the children themselves grow which predicates the maintenance of a euthyroid state. Of particular interest was that children with BMI >2 SD had significantly higher thyroid volume SDS, a similar finding in children exhibiting catch-up growth. This large treatise principally on normality and its definitions is a welcome addition to our knowledge and the literature.

\title{
Clinical trials of $\mathbf{G H}$ At the heart of the matter
}

\section{Subtle alterations of cardiac performance in children with growth hormone deficiency: results of a two-year prospective, case-control study}

\author{
Capalbo D, Lo Vecchio A, Farina V, Spinelli L, Palladino A, Tiano C, Lettiero T, Lombardi G, Colao A, Salerno M \\ Department of Pediatrics, Federico II University of Naples, Naples, Italy \\ J Clin Endocrinol Metab 2009;94:3347-3355
}

Background: Children with GH deficiency (GHD) may have reduced left ventricular (LV) mass, this has not been documented as impairing cardiac function. The aim of this study was to evaluate effects of GHD and GH therapy on cardiac function using load-dependent and load-independent indices of myocardial contractility.

Methods: Echocardiography was performed in 24 GHD children at baseline and at 1 and 2 years after GH therapy and in 24 aged-matched controls with familial short stature.

Results: Compared with controls, GHD children had a had lower left ventricular mass at baseline (left ventricular mass/body surface area $50.6 \pm 1.8$ vs. $60.5 \pm 2.4 \mathrm{~g} / \mathrm{m}^{2} ; \mathrm{p}<0.002$, and left ventricular mass/ height ${ }^{2} 28.7 \pm 1.2$ vs. $\left.33.6 \pm 1.3 \mathrm{~g} / \mathrm{m}^{2} ; \mathrm{p}<0.009\right)$. General systolic function was normal, with fractional shortening showing only a slight trend toward impairment $(34.9 \pm 1.5$ vs. $37.6 \pm 1.1 \%)$. However, subtle left ventricular dysfunction was revealed by load-dependent and load-independent indices of myocardial contractility. There was a lower rate-corrected mean velocity of circumferential fiber shortening in GHD patients compared with controls $(1.0 \pm 0.03$ vs. $1.18 \pm 0.03 \mathrm{circ} / \mathrm{s} ; \mathrm{p}=0.0001)$ and stress shortening index $(0.10 \pm 0.02$ vs. $0.18 \pm 0.02 ; \mathrm{p}<0.007)$ and higher end-systolic stress $(49.2 \pm 1.4$ vs. $\left.45.7 \pm 1.0 \mathrm{~g} / \mathrm{cm}^{2} ; \mathrm{p}<0.05\right)$. There was a significant improvement of cardiac size after 1 year of $\mathrm{GH}$ treatment (left ventricular mass/body surface area $67.8 \pm 2.9 \mathrm{~g} / \mathrm{m}^{2}$; left ventricular mass/height ${ }^{2} 38.2 \pm$ $2.0 \mathrm{~g} / \mathrm{m}^{2} ; \mathrm{p}<0.0001$ and $\mathrm{p}=0.0003$, respectively) and in myocardial contractility (mean velocity of circumferential fiber shortening $1.2 \pm 0.04 \mathrm{circ} / \mathrm{s} ; \mathrm{p}<0.0002$; stress shortening index $0.19 \pm 0.02 ; \mathrm{p}<$ 0.003 ) and reduced afterload (end-systolic stress $43.9 \pm 1.4 \mathrm{~g} / \mathrm{cm}^{2} ; \mathrm{p}<0.03$ ).

Conclusion: This study has demonstrated that GH deficiency is associated with abnormalities in morphology and function in not only adults but also in children and further supports the beneficial effect of GH on the heart.

This is the first study to document that children with GHD have mild alterations in myocardial contractility and impairment in cardiac performance. However, these abnormalities are likely of little actual clinical significance in childhood because general systolic function is within normal limits. This was a case-controlled study, and since children with GHD may have abnormal body composition, with a reduction in lean mass and an increase in body fat, the authors compared the patients with a group of controls who were younger but height and body surface area matched to better compare cardiac parameters. The results of the present study demonstrate that GH therapy is able to reverse all these subtle abnormalities. In addition to a positive effect on cardiac size, 1 year of GH treatment caused a significant decrease in wall stress and a consistent increase in cardiac contractility parameters. The findings are also in agreement with studies of cardiac function in adults with GHD showing similar 
effects but are of shorter duration in children. When atherosclerotic or thrombotic risk factors are considered, GHD was associated with higher serum homocysteine levels compared with controls. Two years of GH treatment significantly decreased homocysteine concentrations and improved lipid profiles with a decrease of total cholesterol and total to HDL cholesterol ratio, compared with both pretreatment and control values. These results suggest that children with untreated GHD may also have subtle abnormalities of their lipid profile that might place them to an increased atherosclerotic or thrombotic risk and on which GH seems to exert a beneficial effect. This study shows that children with severe GHD have a greater impairment of systolic function in addition to a more severe reduction of left ventricular mass when compared with children with partial GHD. However, as children with partial GHD also have a significant reduction in cardiac size compared with controls, myocardial contractility was only slightly impaired and afterload was not significantly increased. GH therapy normalizes cardiac size and function in both severe and partial GHD patients, and with the improvement in atherosclerotic parameters provides yet more evidence of the beneficial effect of GH treatment on the cardiovascular system, which should signal well for long-term health.

\title{
Cardiovascular and metabolic risk profile and acylation-stimulating protein levels in children with Prader-Willi syndrome and effects of growth hormone treatment
}

\author{
de Lind van Wijngaarden RF, Cianflone K, Gao Y, Leunissen RW, Hokken-Koelega AC \\ Dutch Growth Research Foundation, Rotterdam, The Netherlands \\ r.delindvanwijngaarden@erasmusmc.nl \\ J Clin Endocrinol Metab 2010;95:1758-1766
}

Background: The aim of this study was to investigate the metabolic and cardiovascular risk profile and acylation-stimulating protein (ASP) levels and to investigate the effects of GH treatment in children with Prader-Willi syndrome (PWS). ASP stimulates glucose uptake and triglyceride storage in adipose tissue.

Methods: This was a randomized controlled GH trial. 85 children with PWS (mean \pm SD age of $4.9 \pm 3.0$ years) participated in the study. Infants and prepubertal children received GH $\left(1 \mathrm{mg} / \mathrm{m}^{2} / \mathrm{day}\right)$ or served as controls for 12 and 24 months, respectively. Percentage fat was measured (fat \%) with dual-energy $\mathrm{x}$-ray absorptiometry, together with blood pressure, fasting insulin and glucose levels, serum lipids, and ASP levels.

Results: Mean \pm SD fat $\%$ was $28.4 \pm 6.2$ in infants and $36.9 \pm 8.5$ in prepubertal children. $95 \%$ of prepubertal children had a fat $\%$ SD score (SDS) $>2$ SDS. At least one cardiovascular risk factor, defined as hypertension or dyslipidemia, was abnormal in $63 \%$ of infants and $73 \%$ of prepubertal children, with $5 \%$ of all children having the metabolic syndrome. Mean \pm SD baseline ASP was $107 \pm 45 \mathrm{nmol} / \mathrm{l}$ (normal $<58 \mathrm{nmol} / \mathrm{l}$ ) and this correlated with fat mass and TG levels, but GH treatment had no effect on mean ASP levels in this population. GH improved fat $\%$ SDS and the HDLc/LDLc ratio $(\mathrm{p}<0.0001$ and $\mathrm{p}=0.04)$.

Conclusion: A high proportion of children with PWS had dyslipidemia and high ASP levels. Fat $\%$ and high-density lipoprotein cholesterol/low-density lipoprotein cholesterol was improved with GH treatment, but no effect was seen on ASP. High ASP levels may prevent complete normalization of fat $\%$ SDS during GH treatment but may this effect may help to keep glucose and insulin levels within normal ranges.

This RCT of GH treatment confirms that young children with PWS have a high fat mass and dyslipidemia, whereas blood pressure and glucose homeostasis are normal in most patients with only $5 \%$ fulfilling criteria for the diagnosis of metabolic syndrome. GH-treated children showed significant improvement of height, BMI, and IGF-1 during 12 and 24 months of treatment compared with randomized controls. In addition, GH reduced fat mass and fat $\%$ and increased the HDL/LDL ratio. Acylation-stimulating protein (ASP) is produced by adipose tissue and stimulates free fatty acid incorporation into adipose tissue by increasing triglyceride synthesis and storage and by inhibiting hormone-sensitive lipase-mediated triglyceride lipolysis. ASP correlates positively with fat mass and negatively with triglyceride levels. Thus, ASP may contribute to maintaining a high fat\%. The study demonstrated twofold higher ASP levels in young children with PWS, coinciding with an increased fat mass, but normal triglyceride levels. ASP levels were not altered by GH. GH treatment does not 
result in complete normalization of fat mass in children with PWS which suggests that GH insufficiency is not the only factor involved in the mechanism leading to abnormal body composition in PWS. This study provides further evidence for the metabolic benefits of GH treatment in this condition and helps to shed further light on the mechanism of fat accumulation.

\section{New mechanisms \\ Teaching fat-busting}

\section{School-based interventions on childhood obesity: a meta-analysis}

Gonzalez-Suarez C, Worley A, Grimmer-Somers K, Dones V

Centre for Allied Health Evidence, University of South Australia, Adelaide, S.A., Australia

cgsuarez@mnl.ust.edu.ph

Am J Prev Med 2009;37:418-427

Background: Childhood obesity has been recognized as an increasing health problem worldwide and predicts adulthood obesity, which in turn is strongly linked to chronic lifestyle diseases. This meta-analysis set out to evaluate the effectiveness of school-based programs in the prevention and management of childhood obesity.

Methods: A comprehensive literature search for papers to be included for the meta-analysis was undertaken for RCTs and clinical controlled trials published between 1995 and 2007 on school-based interventions that addressed childhood obesity. They were restricted to those in which odds ratios (OR) or standardized mean differences and their $95 \%$ CIs were reported or could be calculated from available data.

Results: The meta-analysis showed that the odds of these school-based intervention programs being significantly protective in the short term for overweight and obese participants was OR $=0.74(95 \%$ CI $0.60,0.92$ ) in comparison with the control arm. Programs that were conducted for more than 1 year had a higher OR of decreasing the prevalence of obesity. However, interventions were not effective in decreasing BMI compared with control treatments, with a weighted mean difference of $-0.62(95 \% \mathrm{CI}$ $=-1.39,0.14)$.

Conclusion: This meta-analysis showed that in the short term, there was convincing evidence that schoolbased interventions can be effective in reducing the prevalence of childhood obesity, but not on reducing BMI. Longer-running programs were more effective than shorter ones.

\section{Effect of school-based physical activity program (KISS) on fitness and adiposity in primary schoolchildren: cluster randomized controlled trial}

Kriemler S, Zahner L, Schindler C, Meyer U, Hartmann T, Hebestreit H, Brunner-La Rocca HP, van Mechelen W, Puder JJ

Institute of Exercise and Health Sciences, University of Basel, Basel, Switzerland

susi.kriemler@unibas.ch

BMJ 2010;340:c2968

Background: This cluster randomized controlled trial aimed to assess the effectiveness of a school-based physical activity program over one school year on physical and psychological health in young schoolchildren.

Methods: 28 classes from 15 elementary schools in Switzerland (540 children, of whom 502 consented and presented at baseline) were randomly selected and assigned in a 4:3 ratio to an intervention $(\mathrm{n}=16)$ or control arm $(\mathrm{n}=12)$ after stratification for grade (first and fifth grade), between August 2005 and June 2006. Children in the intervention arm $(n=297)$ received a multicomponent physical activity program that included structuring the three existing physical education lessons each week and adding two additional lessons a week, daily short activity breaks, and physical activity homework. Children (n $=205$ ) and parents in the control group were not informed of an intervention group. For most outcome measures, the assessors were blinded. The study measures included body fat (sum of four skinfolds), 
aerobic fitness (shuttle run test), physical activity (accelerometry), and quality of life (questionnaires). Secondary outcome measures included body mass index and cardiovascular risk score (average Z score of waist circumference, mean blood pressure, blood glucose, inverted high density lipoprotein cholesterol, and triglycerides).

Results: 498 children in total (mean age 6.9 (SD 0.3) years for first grade, $11.1(0.5)$ years for fifth grade), completed the baseline and follow-up assessments. After adjustment for grade, sex, baseline values, and clustering within classes, in the intervention arm children showed more negative changes in the $\mathrm{Z}$ score of the sum of four skinfolds $(-0.12,95 \% \mathrm{CI}-0.21$ to $-0.03 ; \mathrm{p}=0.009)$ compared with controls. $\mathrm{Z}$ scores for aerobic fitness increased more favorably $(0.17,0.01-0.32 ; \mathrm{p}=0.04)$, as did those for moderate-vigorous physical activity in school $(1.19,0.78-1.60 ; \mathrm{p}<0.001)$, all-day moderate-vigorous physical activity $(0.44,0.05-0.82 ; \mathrm{p}=0.03)$, and total physical activity in school $(0.92,0.35-1.50$; $\mathrm{p}=0.003)$. Z scores for overall daily physical activity $(0.21,-0.21$ to 0.63$)$ and physical quality of life $(0.42,-1.23$ to 2.06$)$ as well as psychological quality of life $(0.59,-0.85$ to 2.03$)$ did not show any significant changes.

Conclusion: A school-based multicomponent physical activity intervention which included some elements that were compulsory improved physical activity and fitness and reduced adiposity in children.

The meta-analysis showed that long-running school-based interventions are effective in preventing childhood overweight and obesity. It found that combined interventions of physical activity in the classroom curriculum were effective in preventing childhood overweight and obesity. The duration of the intervention however was positively associated with its effectiveness. Given these results, the authors suggest that school principals and policymakers should consider implementing school-based interventions as long-term strategies for preventing and managing childhood overweight and obesity. Kreimler et al. presented such an approach in a cluster randomized controlled trial. A multicomponent physical activity intervention delivered during one school year had beneficial effects on physical activity, aerobic fitness, and adiposity and they postulated could thus reduce cardiovascular risk. They concluded that programs with compulsory physical activity components seem to be superior to those based on educational interventions, as adherence is guaranteed. Furthermore, the inclusion of all children in a class avoided any stigmatization of overweight and unfit children and gave all children an equal chance to benefit from this type of intervention. As $90 \%$ of all children and $70 \%$ of the teachers liked the program and wished it to continue, this was very reassuring. The multicomponent and systemic physical activity approach, which included a variety of strategies to enhance physical activity, may have reached more children by broadening the levels of the intervention and the spectrum of activities by making them not only fun but also developing their motor skills too. This approach of integration of increased physical activity into the regular school curriculum going in the long term is the challenge for society.

\section{New uses for old hormones}

\section{Efficacy and safety of oxandrolone in growth hormone-treated girls with Turner syndrome}

Menke LA, Sas TC, de Muinck Keizer-Schrama SM, Zandwijken GR, de Ridder MA, Odink RJ, Jansen M, Delemarre-van de Waal HA, Stokvis-Brantsma WH, Waelkens JJ, Westerlaken C, Reeser HM, van Trotsenburg AS, Gevers EF, van Buuren S, Dejonckere PH, Hokken-Koelega AC, Otten BJ, Wit JM

Department of Pediatrics, Leiden University Medical Center, Leiden, The Netherlands

l.a.menke@lumc.nl

J Clin Endocrinol Metab 2010;95:1151-1160

Background: Although GH therapy has been shown to increase growth and adult height in Turner syndrome (TS), the benefit to risk ratio of adding the weak androgen oxandrolone $(\mathrm{Ox})$ in addition to $\mathrm{GH}$ is unclear.

Methods: A double-blind, randomized, placebo-controlled, dose-response study was performed in 10 centers in The Netherlands. 133 patients with TS were split into three age groups: 1 (2-7.99 years), 2 
(8-11.99 years), or 3 (12-15.99 years). The girls received GH $\left(1.33 \mathrm{mg} / \mathrm{m}^{2} /\right.$ day $)$ from baseline, combined with placebo $(\mathrm{Pl})$ or $\mathrm{Ox}$ in low dosage $(0.03 \mathrm{mg} / \mathrm{kg} /$ day $)$ or conventional dosage $(0.06 \mathrm{mg} / \mathrm{kg} /$ day $)$ from the age of 8 years and estrogens were added from the age of 12 years. Outcome measures included adult height gain (adult height minus predicted adult height) and safety parameters were carefully evaluated.

Results: Compared with $\mathrm{GH}+\mathrm{Pl}, \mathrm{GH}+\mathrm{Ox} 0.03$ increased adult height gain over $\mathrm{GH}+\mathrm{Pl}$ in the intentionto-treat analysis by mean 9.5 SD 4.7 vs. 7.2 SD $4.0 \mathrm{~cm}, \mathrm{p}=0.02$ ) and per-protocol analysis (9.8 SD 4.9 vs. $6.8 \mathrm{SD} 4.4 \mathrm{~cm}, \mathrm{p}=0.02)$. On account of the accelerated bone maturation $(\mathrm{p}<0.001)$, adult height gain on $\mathrm{GH}+\mathrm{Ox} 0.06$ was not significantly different from that on $\mathrm{GH}+\mathrm{Pl}(8.3 \mathrm{SD} 4.7$ vs. $7.2 \mathrm{SD} 4.0 \mathrm{~cm}$, $\mathrm{p}=0.3)$. Breast development was slower on $\mathrm{GH}+\mathrm{Ox}(\mathrm{GH}+\mathrm{Ox} 0.03, \mathrm{p}=0.02 ; \mathrm{GH}+\mathrm{Ox} 0.06, \mathrm{p}=0.05)$, and more girls reported virilization on $\mathrm{GH}+\mathrm{Ox} 0.06$ than on $\mathrm{GH}+\mathrm{Pl}(\mathrm{p}<0.001)$.

Conclusion: In GH-treated girls with TS, the authors discourage the use of the conventional Ox dosage $(0.06 \mathrm{mg} / \mathrm{kg} / \mathrm{day})$ because of its low benefit-to-risk ratio. The addition of Ox $0.03 \mathrm{mg} / \mathrm{kg} / \mathrm{day}$ modestly increases adult height gain and has a fairly good safety profile, except for some deceleration of breast development.

This double-blind, randomized, placebo-controlled, dose-response study of careful design aimed to assess the risk-to-benefit ratio and effectiveness of low and a conventional dose of the weak anabolic steroid in augmentation of the GH induced GH response in girls with TS. Statistical pre-analysis estimated that 15 patients per dosage and age group were needed to achieve a power of $80 \%$ to detect a difference ( $p=0.05$, two-sided) in first-year height velocity of $2 \mathrm{~cm}$ with an assumed SD of 2.6. The eventual group sizes of 39-48 clearly exceeded this. Intention-to-treat analyses were performed and differences in adult height gain were also assessed by a per-protocol analysis. In the per-protocol analysis, adult height gain was $3.1 \mathrm{~cm}$ greater on $\mathrm{GH}+\mathrm{O} \times 0.03$ and $2.2 \mathrm{~cm}$ greater on $\mathrm{GH}+\mathrm{O} \times 0.06$. When correcting for bone age at starting GH therapy, the difference in adult height gain compared with $\mathrm{GH}+$ placebo was $+1.8 \mathrm{~cm}$ on $\mathrm{GH}+\mathrm{O} \times 0.03(\mathrm{p}=0.05)$. Given the lower cost of oxandrolone compared with $\mathrm{GH}$, this result could be seen to produce a greater cost-benefit ratio on improving adult height than $\mathrm{GH}$ alone, and avoids the need to consider the more expensive option of higher GH doses, which may in its own right have other safety considerations. Safety evaluation was a notable feature of this study, with several participants either refusing or stopping conventional dose oxandrolone and the final conclusion advises against this, both on account of a greater virilization effect and also a less good outcome for final height due to acceleration in bone age maturation. Breast development could be slightly delayed but not adversely affected. The lessons and boundaries of the use of this additional agent are clearly spelled out by this study, which provides an excellent evidence base for clinical practice.

\section{Reviews}

\section{Prevalence and risk factors of radiation-induced growth hormone deficiency in childhood cancer survivors: a systematic review}

Mulder RL, Kremer LC, van Santen HM, Ket JL, van Trotsenburg AS, Koning CC, Schouten-van Meeteren AY, Caron HN, Neggers SJ, van Dalen EC

Department of Paediatric Oncology, Emma Children's Hospital/Academic Medical Center, Amsterdam,

The Netherlands r.I.mulder@amc.uva.nl

Cancer Treat Rev 2009;35:616-632

Background: The aim of this systematic review was to evaluate the existing evidence of the prevalence and risk factors of radiation-induced GHD in childhood cancer survivors. Growth hormone deficiency (GHD) is usually the first and commonest endocrine problem occurring after cranial radiotherapy (CRT).

Methods: The authors searched MEDLINE, EMBASE and CENTRAL for studies reporting on radiationinduced GHD in childhood cancer survivors. A meta-regression analysis was performed on the information about study characteristics, prevalence and risk factors abstracted and the quality of each study was assessed. 
Results: Most studies had methodological limitations. The prevalence of radiation-induced GHD was estimated in 33 studies and this varied considerably between 0 and $90.9 \%$. Only three studies had adequate peak GH cut-off limits $(<5 \mu \mathrm{g} / \mathrm{l})$. In these studies the prevalence ranged from 29.0 to $39.1 \%$, with a pooled prevalence of $35.6 \%$. The main risk factors of GHD identified from studies included in this review were higher CRT dose and longer follow-up times. The meta-regression analysis showed that the wide variation in the prevalence of GHD could be explained by differences in maximal CRT dose.

Conclusion: GHD is a frequent consequence after CRT in childhood cancer survivors. The prevalence of radiation-induced GHD ranged from 29.0 to $39.1 \%$ when selecting only studies with adequate peak GH cut-off limits. Higher CRT dose and longer time from treatment are the main risk factors. Additional well-designed studies are needed to estimate the precise prevalence of GHD and to define the exact CRT threshold dose at which GHD occurs.

This systematic review and meta-analysis of factors surrounding the diagnosis of GHD in childhood cancer survivors is helpful if not new, but recommends that future studies should prospectively evaluate all children treated with CRT. Follow-up should be long enough and complete, with precise and accurate outcome definitions and uniform methods of detection. Furthermore, the evolution of different risk factors time should be investigated. The authors also recommend that appropriate multivariate analyses are necessary taking into account the separate and joint effects of CRT and other important prognostic risk factors for GHD. Time to ensure your database is up to date.

\section{Food for thought \\ Swings and roundabouts}

\section{Long-term follow-up of GH-treated girls with Turner syndrome: metabolic consequences}

Bannink EM, van der Palen RL, Mulder PG, de Muinck Keizer-Schrama SM

Department of Pediatrics, Division of Endocrinology, Erasmus MC-Sophia Children's Hospital, Rotterdam,

The Netherlands

Horm Res 2009;71:343-349

Background: This follow-up study of a randomized GH dose-response trial study aimed to investigate the long-term metabolic consequences of childhood GH treatment in young women with Turner syndrome (TS), several years after GH had been discontinued.

Methods: The original trial had three GH dosage arms (1.3, 2.0, and $2.7 \mathrm{mg} / \mathrm{m}^{2} /$ day). 39 TS patients $(20.0 \pm 2.1$ years $)$ participated $4.8 \pm 1.9$ years after finishing $\mathrm{GH}$. The mean $\mathrm{GH}$ treatment duration was $8.7 \pm 2.0$ years. Fasting glucose, insulin, and serum lipids were measured.

Results: For several years after GH discontinuation, insulin sensitivity remained lower, whilst $\beta$-cell function and fasting insulin levels stayed higher than before treatment. Only BMI was proportional to $\beta$-cell function. Serum total cholesterol (TC), low-density lipoprotein and high-density lipoprotein (HDL) were elevated in comparison with values measured 6 months after stopping $\mathrm{GH}$, resulting in higher TC, but also higher HDL levels compared to controls. The atherogenic index remained constant, but below that of the controls.

Conclusion: GH therapy in girls with TS has beneficial effects on serum lipids as well as on growth promotion. Nearly 5 years after discontinuation of GH therapy the favorable effect of GH was still noticeable. The GH-induced decrease in insulin sensitivity, however, did not return to baseline. Whether this was just the natural history of TS is unclear.

Is this good or bad news? This long-term follow-up study, evaluates the metabolic consequences after GH treatment in girls with TS in three dosage schedules, but a no-treatment or placebo arm is not included, so we need to rely on historical data for comparison [6]. Insulin sensitivity remained low, not returning to baseline levels, with, pancreatic $\beta$-cell function impaired and fasting insulin levels remained high. Previously, the authors hypothesized that the higher insulin levels after GH might 
result from a higher BMI, as previous studies had shown a positive correlation between insulin levels and $\mathrm{BMI}$ in normal children and adults and would be in agreement with a previous report on the relationship between the effect of $\mathrm{GH}$ on adiposity and insulin sensitivity on and after GH treatment in TS [7]. A positive association of BMI with $\beta$-cell function, and with higher fasting insulin levels several years after $\mathrm{GH}$ therapy, supports this possible explanation. It is also reported that untreated young TS women have a higher prevalence of insulin resistance and impaired glucose tolerance, so the authors believe the findings in this study may just be part of the natural history of TS [8]. Alternatively, the persistence of low insulin sensitivity and high $\beta$-cell function may be explained by the GH therapy administered. Although no association with $\mathrm{GH}$ dosage or $\mathrm{GH}$ duration is known, such an effect cannot be ruled out. During the first 4 years of GH therapy the study found a decrease in total cholesterol, LDL cholesterol, and atherogenic index, and an increase in HDL and triglycerides. Six months after GH treatment, total cholesterol, LDL and HDL cholesterol increased, resulting in a further decrease in atherogenic index. This pattern extended at long-term follow-up with lipid profiles similar to those reported in untreated TS women being age and BMI independent [6]. This effect is similar to that seen in GHD and treated SGA individuals. So what atherogenic improvements are gained on the swings need to be contrasted with losses in insulin sensitivity on the roundabouts. This is a clear message about the long-term follow-up needed for GH treated individuals.

\section{Follow-up on YB 2009 papers GH and ISS}

\section{High-dose GH treatment limited to the prepubertal period in young children with idiopathic short stature does not increase adult height}

Van Gool SA, Kamp GA, Odink RJ, de Muinck Keizer-Schrama SM, Delemarre-van de Waal HA, Oostdijk W, Wit JM

Department of Pediatrics, Leiden University Medical Center, Leiden, The Netherlands

Eur J Endocrinol 2010;162:653-660

Background: This was a randomized-controlled study to assess the long-term effect of high-dose GH treatment on growth in children with idiopathic short stature (ISS) given in the prepubertal period only.

Methods: 40 prepubertal children aged 4-8 years (girls) or 4-10 years (boys), height SDS $<-2.0$ SDS, and birth length >-2.0 SDS, were randomly allocated to receive $\mathrm{GH}$ at a dose of $2 \mathrm{mg} / \mathrm{m}^{2} /$ day (equivalent to $75 \mu \mathrm{g} / \mathrm{kg} / \mathrm{day}$ at the start and $64 \mu \mathrm{g} / \mathrm{kg} /$ day at the finish) until the onset of puberty for at least 2 years (preceded by two 3-month periods of treatment with low or intermediate doses of GH separated by two washout periods of 3 months) or no treatment as a control group. In 28 cases, adult height (AH) was assessed at a mean (S.D.) age of 20.4 (2.3) years.

Results: The children receiving GH treatment (mean time on high-dose GH 2.3 years; range 1.2-5.0) showed an increased mean height SDS at discontinuation of the treatment of -1.3 (0.8) SDS compared with $-2.6(0.8)$ SDS in the controls. However, bone maturation accelerated significantly in the GH-treated group compared with the controls $(1.6(0.4)$ vs. $1.0(0.2)$ years per year, respectively), associated with an earlier onset puberty. Treatment was not given during puberty in either group and after 3-12 years, AH was $-2.1(0.7)$ and $-1.9(0.6)$ in the GH-treated and control groups respectively. Age was a positive predictor of adult height gain.

Conclusion: High-dose GH treatment given only in the prepubertal period in young ISS children augments height gain during treatment but is associated with an acceleration in bone maturation. This resulted in a similar adult height compared with the untreated controls.

Each year in the Yearbook, outcomes of carefully designed, long-term RCTs of GH treatment are reported as in 2009 there were reports of apparent success [9] and failure [10] of GH treatment in short normal children. Individually they are important, even though conclusions may be different with contrasting conclusions on height gain. Inevitably, as the authors discuss here, results of this study may be limited by small size and dropout over time. Study designs are deliberately different, this one asking separate questions, i.e. What is the effect of GH treatment in ISS children when given a boost in prepuberty only? and Does the dose make a difference? This study makes several innova- 
tions such as examining acceleration in growth of different body proportions (sitting height growth accelerates much faster in the GH-treated group) and a novel technique for expressing pubertal stage in SDS (correcting for age and gender). There was a trend but no statistically significant difference between the groups at Tanner stage 2 . The authors postulate that the most likely explanation is that a high GH dose (approximately three times higher than simple replacement) administered to young children not only leads to faster growth but also results in faster bone maturation. There are few data on GH treatment of young children with ISS, as most other studies started at an average age of 11 years. It seems unlikely that the high-dose per se causes the lack of effect, because children treated with a high GH dose at 11 years achieve an $\mathrm{AH}$ gain of $1.3 \mathrm{SD}$, which is slightly more than that would be gained at a low dose. The authors speculate that the epiphyseal plates of young children could be more sensitive to high doses of GH and/or IGF-1 than at later ages. They state that the results also imply that there may be an inverse $\mathrm{U}$-shaped relationship between $\mathrm{GH}$ dose and $\mathrm{AH}$ gain if treatment is started at a young age. Standard dose $\mathrm{GH}$ is positively associated with $\mathrm{AH}$, but in young children higher doses may decrease $\mathrm{AH}$ gain due to accelerated maturation of the epiphyseal plates and possibly also of the GnRH regulatory centre with the effect on growth having reached a plateau. A disappointing result from a carefully crafted study, but the discussion (as alluded to above) fosters interesting hypotheses as to the relationships between $\mathrm{GH}$ and maturation.

Acknowledgement

I would like to thank Dr. Stephen O'Riordan of the Children's Hospital, Leicester Royal Infirmary, for assistance with the selection of some papers for review.

\section{References}

1. O'Riordan SM, Butler G: Evidence-based medicine in pediatric endocrinology; in Carel JC, Hochberg Z (eds): Yearbook of Pediatric Endocrinology. Basel, Karger 2009, pp 181-197.

2. NICE Technology Appraisal Guidance 151: Continuous subcutaneous insulin infusion for the treatment of diabetes mellitus (review of technology appraisal guidance 57) 2008. http://guidance.nice.org.uk/TA151/Guidance/pdf/English

3. Butler GE: Assessment of growth and puberty; in Ranke MB, Price DA, Reiter EO (eds): Treatment with Recombinant Human Growth Hormone in Children and Adolescence - 20 Years of KIGS. Basel, Karger 2007, chapt 2, pp 6-15.

4. Rosenfeld RG, Albertsson-Wikland K, Cassorla F, Frasier SD, Hasegawa Y, Hintz RL, LaFranchi S, Lippe BM, Loriaux DL, Melmed S, Preece MA, Ranke MB, Reiter EO, Rogol AD, Underwood LE, Werther GA: Diagnostic controversy: the diagnosis of childhood growth hormone deficiency revisited. J Clin Endocrinol Metab 1995;80:1532-1540.

5. Ho KK: Consensus guidelines for the diagnosis and treatment of adults with GH deficiency. II. A statement of the GH Research Society in association with the European Society for Pediatric Endocrinology, Lawson Wilkins Society, European Society of Endocrinology, Japan Endocrine Society, and Endocrine Society of Australia. Eur J Endocrinol 2007;157:695700.

6. Caprio S, Boulware S, Diamond M, Sherwin RS, Carpenter TO, Rubin K, et al: Insulin resistance: an early metabolic defect of Turner's syndrome. J Clin Endocrinol Metab 1991;72:832-836.

7. Wooten N, Bakalov VK, Hill S, Bondy CA: Reduced abdominal adiposity and improved glucose tolerance in growth hormone-treated girls with Turner syndrome. J Clin Endocrinol Metab 2008;93:2109-2114.

8. Sagi L, Zuckerman-Levin N, Gawlik A, Ghizzoni L, Buyukgebiz A, Rakover Y, et al: Clinical significance of the parental origin of the X chromosome in Turner syndrome. J Clin Endocrinol Metab 2007;92:846-852.

9. Albertsson-Wikland K, Aronson AS, Gustaffson J, Hagenas L, Ivarsson SA et al: Dose-dependent effect of growth hormone on final height in children with short stature without growth hormone deficiency. J Clin Endocrinol Metab 2008; 93:4342-4350.

10. Elder CJ, Barton JS, Brook CG, Preece MA, Dattani MT, Hindmarsh PC: A randomised study of the effect of two doses of biosynthetic human growth hormone on final height of children with familial short stature. Horm Res 2008;70:8992. 